[ESTUDIOS SOBRE EL IDEALISMO ALEMÁN]

\title{
Entre iusnaturalismo y positivismo jurídico. La doctrina del derecho de Fichte de 1796
}

\author{
Between natural law and legal positivism \\ The Fichte's doctrine of right of 1796 \\ OSCAR CUBO \\ Universidad de Tréveris (Alemania)
}

Recibido: 24-12-2013 Aprobado definitivamente: 21-03-2014

\begin{abstract}
RESUMEN
La filosofía del derecho de Fichte supone una importante anomalía dentro del Idealismo Alemán, en especial por su problemático posicionamiento en relación con la filosofía del derecho de Kant y su reelaboración del derecho natural. Fichte publica el Fundamento del Derecho Natural según los principios de la Doctrina de la Ciencia en 1796, es decir, un año después de la publicación de Hacia la paz perpetua y un año antes de la publicación de los «Principios metafísicos de la doctrina del derecho» de la Metafísica de las Costumbres. La formulación y fundamentación que lleva a cabo Fichte en esta obra del derecho natural es enteramente original y a la vez internamente problemática debido a la tensión que en ella se da entre la validez normativa de los principios del derecho y la realización positiva de los mismos. Ejemplo paradigmático de esta tensión lo ofrece la noción de «derecho originario» (Urrecht) que Fichte elabora a lo largo de su trabajo de 1796. Nuestra investigación se va a centrar en analizar esta noción de «derecho originario» en la doctrina del derecho de Fichte para desplegar una interpretación iusnaturalista de la misma con vistas a relativizar o por lo menos matizar las lecturas preeminentemente positivistas de la filosofía del derecho de Fichte.

\section{PALABRAS CLAVES}

IDEALISMO ALEMÁN, DERECHO NATURAL, PRINCIPIOS DEL DERECHO Y DERECHO ORIGINARIO
\end{abstract}

Suplemento 19 (2014)

(C) Contrastes. Revista internacional de filosofía, pp. 13-26. ISSN: 1136-9922

Departamento de filosofía, Facultad de Filosofía y Letras, Universidad de Málaga Campus de Teatinos, E-29071 Málaga (España) 


\begin{abstract}
Fichte's philosophy of right constitutes an anomaly within German Idealism, particularly due to its problematic positioning in relation to Kant's philosophy of right and his reworking of natural right. Fichte published Foundations of Natural Right in Accordance with the Principles of the Doctrine of Science in 1796, that is, a year after the publication of Towards Perpetual Peace and a year before the publication of «Metaphysical Principles of the Doctrine of Right», in Metaphysics of Morals. Fichte's formulation and substantiation in this work on natural right is entirely original and, at the same time, internally problematic due to the tension created between the regulatory validity of the principles of right and their positive realisation. A paradigmatic example of this tension can be seen in the notion of «primordial right» (Urrecht) developed by Fichte throughout his 1796 work. Our research will centre on analysing the notion of «primordial right» in Fichte's Doctrine of Right to present a natural law-based interpretation of this right with a view to qualifying or at least placing into perspective the pre-eminently positivist readings of Fichte's philosophy of right.
\end{abstract}

\title{
KEYWORDS \\ GERMAN IDEALISM, NATURAL RIGHT, PRINCIPLES OF RIGHT, PRIMORDIAL RIGHT
}

\section{LA COMPRENSIÓN DE FICHTE DEL DERECHO NATURAL}

LA FILOSOFÍA DEL DERECHO DE FICHTE continúa la reformulación racional del derecho natural emprendida por Kant en 1793 en Del tópico: «tal vez esto sea correcto en la teoría, pero no sirve para la práctica» y en Hacia la paz perpetua de 1795. Esta reformulación se caracteriza por dejar a un lado los presupuestos antropológico-metafísicos del antiguo derecho natural y reformular sus premisas suprapositivas en términos puramente racionales. En el antiguo derecho natural estos principios abarcan las normas jurídicas suprapositivas que están por encima de la legislación humana y cuya validez no depende de ningún legislador humano. Ejemplos paradigmáticos de esta concepción del derecho natural lo ofrecen Aristóteles y su fundamentación del derecho natural a partir de la naturaleza del hombre, o San Agustín y Santo Tomás y su fundamentación del derecho natural en la voluntad suprema de Dios. La rehabilitación del derecho natural en Kant y en Fichte reformula este punto de vista iusnaturalista con vista a esclarecer y establecer los principios racionales del derecho que en tanto que principios de todo ordenamiento jurídico poseen una validez suprapositiva y supraestatal; es decir, al igual que Kant, Fichte entiende el derecho natural como una disciplina fundada en la razón y emancipada de los presupuestos metafísicos del derecho natural antiguo. ${ }^{1}$

\footnotetext{
1 Cfr., GA. I/3, 319.
} 
La doctrina del derecho de Fichte de 1796 descansa en dos premisas normativas fundamentales: la primera de estas premisas es, por decirlo en términos kantianos, la ley universal del derecho ${ }^{2}$ y la segunda, su teoría de los derechos originarios. Ambas premisas tienen su sede en la razón práctica y son presentadas por Fichte como premisas universales del derecho racional. La ley universal del derecho expresa el principio por el cual «cada miembro de la sociedad se deja limitar su propia libertad externa mediante la libertad interior, de tal modo que también puedan ser externamente libres todos los otros junto a él». ${ }^{3}$ Este principio descansa en una limitación igualitaria del uso de la libertad de los miembros de una determinada comunidad y como en el caso de Kant ofrece el pilar jurídico elemental de la coexistencia entre seres libres y racionales. Entendidas y extraídas a partir de este principio universal del derecho, las condiciones de la universalidad, igualdad y reciprocidad jurídicas constituyen elementos normativos fundamentales para todo ordenamiento jurídico positivo.

La segunda premisa normativa del Fundamento del Derecho Natural es la noción de derecho originario (Urrecht). Esta noción se funda dentro del sistema de la doctrina de la ciencia en el concepto de una personalidad libre y es indisociable del mismo. Todo sujeto singular en virtud de su personalidad es portador originario de ciertos derechos. Pero ¿de qué derechos? Fichte ofrece una definición general de derecho originario a partir de la cual lleva a cabo una triple concretización del mismo. La definición general de derecho originario es la siguiente: «el derecho originario es el derecho absoluto de la persona a ser en el mundo sensible únicamente la causa (y de no ser, por tanto, nunca algo causado (Bewirktes))». ${ }^{4}$ Este derecho como su propio nombre indica no es un derecho adquirido, sino un derecho innato a cada persona. Esto significa que la validez de este derecho originario no depende de ningún acto jurídico par8ticular, de modo que tampoco puede ser enajenado, ni otorgado por medio de ningún ordenamiento jurídico. Es más, al no poder ser adquirido ni suprimido por medio de ningún contrato positivo, este derecho constituye el marco normativo de todos los contratos que hayan de tener lugar en un sistema jurídico positivo. En contraste con los derechos originarios el resto de derechos que poseen los seres humanos tienen que ser adquiridos por medio de un contrato y ser fijados por medio de una legislación positiva.

2 Cfr., Kant MdS VI, 231: «la ley universal del derecho [reza]: obra externamente de tal modo que el uso libre de tu arbitrio pueda coexistir con la libertad de cada uno según una ley universal»

3 GA. I/3, 320 .

4 GA. I/3, 404. La definición que da Kant un año después del único derecho innato del hombre es la siguiente: «la libertad (independencia con respecto al arbitrio de otro), en la medida en que puede coexistir con la libertad de cualquier otro, según una ley universal, es este derecho único, originario, que corresponde a todo hombre en virtud de su humanidad» MdS VI, 237. 
Fichte formula esta idea en los siguientes términos: el derecho originario de los seres humanos en tanto que seres racionales y libres «precede a todos los contratos jurídicos y únicamente él los hace posibles; [es] el derecho a presuponer que todos los hombres pueden establecer con él, mediante contratos, una relación jurídica. Sólo éste constituye el verdadero derecho del hombre, que corresponde al hombre como hombre: la posibilidad del adquirir derechos». ${ }^{5}$ Fichte coincide con Kant en que pretender enajenar la propia libertad por medio de un contrato supone una contradicción performativa, puesto que a través de esta enajenación aquel que firmara el contrato dejaría de estar en condiciones de poder firmarlo. ${ }^{6}$ Dicho de otro modo, los derechos originarios son derechos inalienables de la persona, por lo que ninguna persona puede prescindir de ellos sin suprimir al mismo tiempo su propia personalidad. Por este motivo, el derecho originario de la persona es un derecho constitutivo de todo ser humano que debe estar garantizado en todo ordenamiento jurídico positivo al estar a la base de todo posible contrato incluido también cualquier «contrato social».

Ahora bien, esta definición general del derecho originario tiene que ser especificada, según Fichte, por medio del principio universal del derecho. En efecto, la formulación abstracta del derecho originario de los seres humanos no contiene ninguna prescripción por lo que respecta al uso externo de la propia libertad frente a otros seres finitos igualmente libres y racionales, de modo que este derecho tomado al pié de la letra supondría un derecho ilimitado a la libertad por parte de todos y cada uno de ellos; es decir, tomado en su literalidad el derecho a ser únicamente causa en el mundo sensible y nunca algo causado impide toda interacción externa entre distintos seres libres y racionales. Dicho de otro modo, la formulación del derecho originario tomada en su literalidad hace imposible el disfrute de semejante derecho en presencia de dos o más seres racionales, ya que hace imposible la coexistencia entre los sujetos que pretendan ser portadores por igual de dicho derecho. Si este derecho originario no quedase limitado por la ley universal del derecho, el uso de la libertad de los distintos sujetos se contradeciría entre sí en virtud del derecho absolutamente ilimitado de ser sólo causa y nunca efecto en el mundo de los fenómenos. ${ }^{7}$

La absolutización de este derecho originario sin referencia alguna al principio del derecho desemboca en un derecho contradictorio e irrealizable, puesto que no puede poner un límite originario al uso de la libertad de las personas entre sí en el mundo sensible. Sólo el principio del derecho está en condiciones, pues, de trazar una distinción entre el uso legítimo e ilegítimo de la libertad

5 GA. I/3, 163

6 Cfr., Kant MdS VI, 283: «un contrato por el que una parte renuncia a su entera libertad en beneficio del otro, por tanto, deja de ser persona [es en sí mismo contradictorio, porque] deja de ser persona, y consecuentemente tampoco tiene que cumplir el contrato».

7 Cfr., GA. I/3, 411. 
externa de cada uno, límite que se ha de incorporar a la definición general del derecho originario del hombre. Conjugados ambos principios normativos el resultado es que los portadores de este derecho originario a la libertad no pueden lesionar tampoco en términos absolutos este mismo derecho en los demás. ${ }^{8}$ Este derecho originario ha de limitarse como condición indispensable de su propia realización a los criterios de la universalidad, la igualdad y la simétrica limitación del uso propio de la libertad para que todos los sujetos puedan disfrutar en el mismo grado y medida del mismo espacio de libertad.

Bajo esta restricción, el derecho originario a la libertad significa en términos generales el derecho a la no injerencia ajena en el uso propio de la libertad, pero teniendo en cuenta que este uso de la libertad resulta contradictorio cuando se lleva a cabo en un escenario social en el que interactúan distintos seres libres y racionales. En este escenario interactivo los individuos no pueden ver asegurado su derecho originario sin limitar al mismo tiempo este derecho con arreglo a los criterios de la simetría y la reciprocidad. La deficiencia de la formulación general del derecho originario es, pues, que no contiene ninguna especificación para su ejercicio en la interacción con los demás. Esta especificación la obtiene, empero, gracias a la ley universal del derecho que supone una limitación del mismo como condición para su propia realización.

El derecho originario a la libertad ha de conformarse, pues, a la condición normativa que expresa el principio del derecho, según la cual este uso de la libertad tiene que ser compatible con el uso de la libertad de los demás. De este modo, obtiene la definición del derecho originario la primera de sus tres especificaciones dentro de la doctrina del derecho de Fichte. En virtud de esta primera especificación, ninguna persona tiene derecho a ejercer una acción en el mundo sensible que haga imposible o restringa arbitrariamente la libertad de los demás. ${ }^{9}$ De ello se sigue una segunda especificación, a saber, que cada sujeto tiene el derecho originario a su propio cuerpo en tanto que el cuerpo propio es el representante sensible de la personalidad de los sujetos, y una tercera especificación, el derecho originario a ejercer una influencia continuada en el mundo sensible para lo que se requiere un derecho de propiedad sobre cosas exteriores al propio cuerpo.

Detengámonos brevemente en cada una de estas especificaciones. Por lo que respecta a la segunda especificación, esto es, al derecho originario al propio cuerpo, Fichte considera que este derecho al propio cuerpo como condición material de la libertad es al mismo tiempo el derecho que tienen todos los seres libres y racionales a la inviolabilidad de su cuerpo en tanto que representante sensible de su personalidad; y por lo que respecta a la tercera especificación de este derecho originario, a saber, el derecho a ejercer una influencia continuada

8 Cfr., Thiele 2002: 22.

9 Cfr., Papadopoulos 1993: 112. 
en el mundo sensible conforme al principio universal del derecho, lo que implica, según Fichte, es el derecho originario a la propiedad no sólo de uno mismo, sino a poder tener en propiedad cosas exteriores al propio cuerpo en tanto que condición igualmente material del ejercicio de la propia libertad. En efecto, para poder ejercer una influencia continuada en el mundo sensible con arreglo a su libertad, las personas necesitan poder disponer de cosas bajo la forma de la propiedad, esto es, más allá de la mera posesión empírica de las mismas siempre incierta y provisional. ${ }^{10}$ Todo lo cual ofrece el presupuesto normativo para la construcción del «derecho privado» que en último término y al igual que en la doctrina del derecho de Kant se funda en el mencionado derecho originario de la libertad.

Pues bien, por medio de estas tres especificaciones del derecho originario obtenemos el perfil normativo de la doctrina del derecho de Fichte de 1796 que podemos resumir del siguiente modo: en primer lugar, el derecho originario de cada individuo a una libertad igualitaria dentro de una comunidad jurídica, en segundo lugar, el derecho a la posesión inalienable del propio cuerpo, que implica el derecho a la inviolabilidad del propio cuerpo y, por último, el derecho originario a la propiedad privada como condición material para el uso continuado de la libertad en el mundo de los fenómenos. Ahora bien, estos tres «derechos fundamentales» en tanto que principios normativos del marco constitucional de todo ordenamiento positivo requieren, según Fichte, de un acto constituyente, esto es, de un acto de reconocimiento intersubjetivo para dejar de ser meras proclamaciones normativas y pasar a ser realidades jurídicas dentro de un ordenamiento positivo.

\section{EL RECONOCIMIENTO INTERSUBJETIVO DE LOS DERECHOS ORIGINARIOS}

En la doctrina del derecho de 1796 Fichte se muestra muy crítico con las interpretaciones iusnaturalistas de los derechos originarios que se complacen con proclamarlos como derechos humanos originarios ajenos y por encima de toda legislación positiva. Por el contrario, Fichte considera que el contenido normativo de los derechos originarios no es suficiente para proclamarlos como «derechos» en términos jurídicos. Fuera de todo acto intersubjetivo de reconocimiento, esto es, fuera de su positivación jurídica, los derechos originarios son una ficción jurídica sin realidad alguna. ${ }^{11}$ Según Fichte, los derechos originarios necesitan de su reconocimiento intersubjetivo para obtener una validez jurídica, puesto que «toda relación jurídica entre personas determinadas está condicionada por su reconocimiento mutuo». ${ }^{12}$

10 Para esta distinción en Kant, cfr., MdS VI, 257ss.

$11 \mathrm{Cfr}$., GA I/3, 112.

12 GA I/3, 413. Fichte se pronuncia en los siguientes términos: «no existe, por consiguiente, ningún derecho natural, en el sentido que a menudo se ha tomado el término, es decir, 
Para el establecimiento de relaciones jurídicas se requiere un querer común, ya que «nadie, por su mera voluntad, puede realizar una tal comunidad con otro si éste no tiene la misma voluntad y si, en virtud de ella, no se somete a la ley jurídica». ${ }^{13}$ La validez hipotética de los principios normativos del derecho se puede, pues, formular del siguiente modo: si alguien quiere entrar en una situación jurídica con otro ser libre y racional «entonces tiene que querer también la ley del derecho; sólo tras la decisión a favor de dicha socialización obtiene el principio del derecho su validez práctica». ${ }^{14}$ La positivación del derecho originario a la libertad requiere, pues, que «cada miembro de la sociedad se deje limitar su propia libertad externa mediante la libertad interior, de tal suerte que también puedan ser externamente libres todos los otros junto a él». ${ }^{15}$

A la base de la necesidad de un reconocimiento intersubjetivo de los derechos originarios está, pues, la idea de que toda relación jurídica presupone una relación interpersonal entre distintos individuos, por lo que en sentido estricto «individuos aislados fuera de cualquier contexto no pueden poseer derecho, y tampoco derechos originarios o derechos naturales $\gg .{ }^{16}$ Dicho de otro modo, para Fichte, no hay ninguna relación jurídica ni tampoco ninguna comunidad jurídica sin el reconocimiento recíproco de los derechos naturales y el mutuo reconocimiento de la libertad originaria de las personas. ${ }^{17}$ Toda comunidad jurídica requiere, pues, un ejercicio de reconocimiento para el establecimiento de relaciones jurídicas entre los individuos. A partir de este reconocimiento fundamental se pueden desarrollar paulatinamente diversos contratos entre los mismos hasta configurar por entero un ordenamiento jurídico positivo.

La necesidad jurídica de un ejercicio intersubjetivo de reconocimiento implica para Fichte que el establecimiento de un estado civil es el resultado contingente de un acto de reconocimiento multilateral por parte de un grupo de individuos; es decir, como la instauración de una comunidad jurídica depende del arbitrio de sus miembros, el establecimiento de un ordenamiento jurídicopositivo ha de tomarse como el resultado de una decisión de carácter técnicopragmática por parte de cada uno de ellos. Dicho de otro modo, no se puede «mostrar ningún motivo absoluto por el que alguien debería erigir en ley de su voluntad y de sus acciones la fórmula del derecho: «limita tu libertad de tal ma-

no es posible ninguna relación jurídica entre hombres, salvo en una res publica y bajo leyes positivas. [...] Pero lo que perdemos por un lado, lo recuperamos del otro incluso con ganancias; pues el Estado mismo se convierte en el estado natural del hombre, y sus leyes no deben ser ninguna otra cosa salvo el derecho natural realizado (als das realisierte Naturrecht)» GA $\mathrm{I} / 3,432$.

$13 \mathrm{GA} \mathrm{I} / 3,387$.

14 Kersting 2007: 136.

$15 \mathrm{GA} \mathrm{I} / 3,320$.

16 Thiele 2002: 13 .

17 Cfr. Papadopoulos 1993: 112.

Suplemento 19 (2014) de Contrastes. Revista internacional de filosofía 
nera que también el otro pueda ser libre a tu lado». Se puede comprender de todos modos que no puede existir ninguna comunidad de seres libres como tales si cada uno no está sometido a esta ley, y que, por consiguiente, quien quiera esta comunidad necesariamente tiene que querer también la ley. Por consiguiente, esta ley tiene validez hipotética. Si una comunidad de seres libres como tales debe ser posible, entonces tiene que valer la ley jurídica». ${ }^{18}$ Este acto de sometimiento a la ley jurídica tiene, pues, una necesidad hipotética, o si se quiere, una necesidad condicionada a la voluntad individual de aquellos que han de someterse a la misma.

Este sometimiento voluntario es conditio sine qua non para que los derechos originarios de las personas se conviertan en derechos positivos, esto es, en derechos fundamentales dentro de un ordenamiento jurídico positivo. A nivel conceptual esto permite diferenciar una fase prejurídica (o proceso constituyente) en la que se tienen que reconocer dichos derechos para que pasen a formar parte del derecho positivo, y una fase jurídica en la que quedan establecidos dichos derechos como horizonte jurídico de toda posible interacción entre los individuos. Sin embargo, y está es una de las mayores dificultades de la doctrina del derecho de Fichte de 1796, el primero de estos escenarios, a saber, el escenario constituyente del ordenamiento jurídico está atravesado por una inestabilidad estructural, ya que el reconocimiento de los derechos de los demás exige un sometimiento unilateral de cada individuo a la ley jurídica sin que haya garantía alguna de que los demás también se someterán a la misma. Es decir, bajo el reconocimiento nominal de los derechos de los demás, que siempre tiene que ser nominal en un escenario prejurídico, nadie puede tener la seguridad «de que el otro limitará su libertad como yo la mía». ${ }^{19}$

Esta falta de garantía es a su vez el indicador de que dicho reconocimiento como condición previa para el establecimiento de relaciones jurídicas tiene lugar en un estado de naturaleza prejurídico, y muestra que este ejercicio de reconocimiento es una condición necesaria, pero no suficiente, para el establecimiento de un orden legal. El reconocimiento intersubjetivo de los derechos es, ciertamente, la manifestación de una voluntad que quiere abandonar este estado de naturaleza e implica el primer paso para su superación. Pero querer superar este estado de naturaleza jurídico, esto es, querer someterse al derecho y querer someterse a las leyes jurídicas todavía no es suficiente para el establecimiento de un ordenamiento jurídico positivo, puesto que el reconocimiento unilateral del derecho de los demás no contiene ninguna garantía de que los demás se atengan de hecho al mismo principio jurídico.

$18 \mathrm{GA} \mathrm{I} / 3,387$.

19 Papadopoulos 1993: 129.

Suplemento 19 (2014) de Contrastes. Revista internacional de filosofía 


\section{LA INSTAURACIÓN ESTATAL DEL DERECHO POSITIVO}

El abandono del estado de naturaleza requiere, según Fichte, el reconocimiento omnilateral de todos los individuos de querer someterse a leyes jurídica. Este acto de reconocimiento es una condición indispensable para la positivización y concretización de los derechos originarios en un sistema jurídico. Sin embargo, este acto de reconocimiento posee una estructura enteramente dilemática, conocida en la «teoría de juegos», como estructura del «dilema del prisionero». En efecto, la estructura dilemática de este acto de reconocimiento recíproco reside en que para el establecimiento de relaciones jurídicas es necesario que todos (sin excepción) se involucren en este acto de reconocimiento. Este acto de reconocimiento presupone, en primer lugar, que cada individuo entiende que la renuncia recíproca y simétrica a un mismo espacio de libertad es una estrategia alternativa y más ventajosa al derecho del uso completo de la libertad que supone el conflicto de todos contra todos. En segundo lugar, que esta alternativa sólo resulta más ventajosa si todos los demás renuncian a dicha libertad. Ahora bien, y aquí reside el problema, la mera posibilidad o falta de garantía de que algunos no se atengan realmente a dicho reconocimiento y adopten una actitud parasitaria frente a los demás, hace que la opción más ventajosa sea la de no renunciar al uso completo de la propia libertad, mientras que no haya garantías de que los demás también vayan a limitar su libertad con arreglo al mismo principio, aunque ello reconduzca la situación al punto suboptimal del conflicto de todos contra todos.

Bajo el riesgo de que los demás no renuncien realmente al uso ilimitado de su libertad nadie se someterá racionalmente al principio universal del derecho mientras que no haya garantías de que también los demás lo harán. La desconfianza generalizada de que los demás vayan a auto-limitar su libertad es, pues, lo que condena al fracaso este escenario de reconocimiento intersubjetivo, mientras no haya garantía alguna para su cumplimiento. Esta situación obliga, pues, a los habitantes del estado de naturaleza a permanecer en dicho estado incluso en contra de su voluntad de querer instaurar un estado jurídico positivo.

La salida del estado de naturaleza depende, pues, únicamente de que haya una instancia objetiva que asegure el cumplimiento de dicho reconocimiento por medios coactivos; es decir, la salida del estado de naturaleza depende en términos generales del establecimiento de una autoridad jurídica y legislativa reconocida universalmente con capacidad coactiva, que permita resolver de una manera objetiva los litigios jurídicos por medio de una fijación clara del contenido de los derechos originarios de cada uno de sus miembros. Es decir, dos son los efectos fundamentales del establecimiento de las relaciones jurídicas entre los hombres, en primer lugar, salvar la situación dilemática del reconocimiento recíproco entre los individuos y, en segundo lugar, dar un contenido concreto y específico a cada uno de los derechos originarios anteriormente mencionados. 
Analicemos el primero de estos efectos. La instauración de una instancia jurídica coactiva permite superar la desconfianza del estado de naturaleza más allá de la declaración moral de intenciones de cada uno de sus miembros. Dicho de otro modo, ninguna declaración de buenas intenciones es suficiente «para dar estabilidad y consistencia a la relación legal. En efecto, aunque reinaran la buena fe y la confianza, basta con una sola transgresión de alguna de las personas, para que la confianza mutua se pierda irremediablemente». ${ }^{20}$ En este sentido, el Estado como instancia controladora y sancionadora de las leyes genera entre sus miembros la confianza de que los demás cumplirán con lo prometido, obligando a todos a través de las amenazas de sanciones a cumplir con las leyes «como si tuvieran una voluntad firme e inquebrantable de cumplir [con las mismas], aunque de hecho no la tengan» ${ }^{21}$ Este sistema de sanciones tiene como objetivo hacer que no valga la pena infringir las leyes para nadie de los que están sometidos a ellas. De este modo el sistema coactivo jurídico del Estado puede garantizar la confianza en el cumplimiento generalizado de las leyes, al acompañar su quebrantamiento con fuertes sanciones penales.

La desmoralización del derecho natural en la doctrina del derecho de Fichte resulta patente cuando afirma que la «buena voluntad» asociada a este ejercicio de reconocimiento mutuo no basta para dar estabilidad y seguridad a las relaciones jurídicas, ni tampoco para abandonar el estado de naturaleza jurídico del que no se puede salir sin instaurar un Estado y un poder legal coactivo que garantice el cumplimiento de las leyes por medio de sanciones jurídicas. Sólo así puede neutralizarse de forma efectiva la desconfianza propia del estado de naturaleza, a saber, haciendo que la violación de las reglas no valga la pena. El establecimiento de un ordenamiento jurídico estatal resuelve, pues, «la tensión entre la ventaja a corto plazo del rompimiento de la norma y la ventaja a largo plazo de la validez de las normas y enlaza el autointerés a corto plazo con la el autointerés a largo plazo». ${ }^{22}$ Es decir, «el delincuente deberá calcular las consecuencias de su acción desde su autointerés, y concluirá que la realización del crimen le traerá más desventajas que ventajas. De modo que el ciudadano cumplirá con la ley $[\ldots]$ por mero egoísmo racional» ${ }^{23}$ y se comportará como si fuera un ciudadano plenamente moral.

Por medio de este dispositivo se obtiene que de cada acción ilegal resulte lo contrario de lo perseguido y así se fuerza a que todos los miembros de una comunidad jurídica quieran sólo lo que es conforme al derecho; o como dice Fichte, la instauración de «un dispositivo como el descrito significa [instaurar] una

20 Arrese 2010: 83.

21 Arrese 2010: 84.

22 Kersting 2001: 102.

23 Arrese 2010: 85.

Suplemento 19 (2014) de Contrastes. Revista internacional de filosofía 
ley de coacción (Zwangsgesetz)» ${ }^{24}$ que desmotive al criminal de buscar su propio provecho a costa de los demás. Dicho en términos positivos: por medio de sus sanciones jurídicas el Estado ofrece un motivo racional para que se cumplan las leyes, haciendo que su seguimiento sea algo incardinado en el autointerés a corto plazo de cada uno de los que están sometidos a ellas. El establecimiento de un poder coactivo es, por tanto, lo que hace que resulte de interés general sujetarse a las normas y el único modo de salvar la estructura dilemática del estado de naturaleza jurídico. ${ }^{25}$

No menos importante es igualmente el segundo efecto del establecimiento de un ordenamiento jurídico, a saber, el de convertir en derechos positivos a los derechos originarios que sin esta positivación jurídica no son sino meras ficciones normativas sin valor jurídico alguno. Esta positivación concierne a los tres elementos fundamentales del derecho originario de todo ser libre y racional, esto es, al derecho a la libertad, al derecho de auto-propiedad y al derecho a la propiedad privada de cosas exteriores del mundo. Así, con anterioridad a su positivación jurídica, el derecho a la libertad es «meramente formal y no determina ninguna cantidad; sólo pone el qué (das Dass), pero no el cuánto (das Inwieweit)» ${ }^{26}$ esta última determinación sólo acontece con la positivación del mismo, ya que en virtud de su positivación se determina «el cuánto» de dicha libertad de una manera concreta y precisa. Por medio de la positivación del derecho se establece «qué cantidad de libertad corresponde a cada individuo según el principio del derecho ${ }^{27}$ y se elimina con ello la incertidumbre cognoscitiva del estado de naturaleza jurídico que se caracteriza por no contener ninguna prescripción clara y positiva acerca del «cuánto» en cuestión; es decir, la positivación del derecho originario a la libertad da a conocer los límites exactos del uso de su libertad de todos los ciudadanos en su interacción con los demás dentro de un ordenamiento jurídico concreto.

Lo mismo sucede con la positivación del derecho originario a la propiedad de uno mismo y del derecho a poseer cosas exteriores en el mundo. Ciertamente, «toda propiedad se funda en el reconocimiento recíproco y éste está condicionado por una declaración recíproca», ${ }^{28}$ pero esta declaración sólo obtiene su garantía y su clara delimitación con la instauración de un ordenamiento jurídico positivo. Dicho de otro modo, aún cuando este reconocimiento tenga lugar en el estado de naturaleza jurídico y cada uno se declare dispuesto a respetar la propiedad de los demás, en este estado de naturaleza nadie puede estar seguro de no estar lesionando los derechos del otro, porque en este escenario no hay criterios

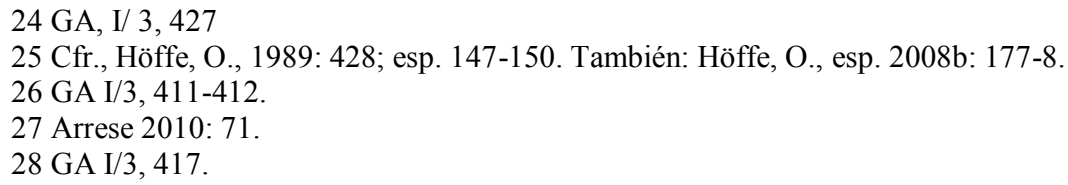


objetivos para detentar la propiedad de un objeto más allá de la presunción de que no sea la propiedad de ningún otro. ${ }^{29}$ La positivación del «derecho privado» permite, pues, no sólo garantizar dicha promesa por medio de coacciones legales, sino también eliminar esta incertidumbre cognoscitiva característica del estado de naturaleza jurídico.

En definitiva, la positivación jurídica de los derechos originarios (de la libertad y la propiedad) permite dar un contorno preciso a los mismos dentro de un ordenamiento jurídico y superar la estructura dilemática del reconocimiento de estos mismos derechos en el estado de naturaleza jurídico. La diferencia fundamental entre este estado de naturaleza y el estado civil reside, pues, en que «la posibilidad de la relación jurídica entre personas en el ámbito del derecho natural está condicionada por la confianza y la fe recíproca», ${ }^{30}$ mientras que en el estado civil estas relaciones se basan en el poder coactivo del Estado como garante último del cumplimiento del derecho positivo. ${ }^{31}$

\section{CONCLUSIÓN: PROPUESTA INTERPRETATIVA}

A través de los pasos que hemos seguido, esto es, siguiendo el hilo argumental de la reformulación fichteana del derecho natural como derecho racional, su compresión de los derechos originarios del hombre, y su necesidad de convertirlos en derechos jurídicos positivos puede llevarse a cabo una lectura preeminentemente normativa del Fundamento del Derecho Natural de 1796. Ciertamente, Fichte enfatiza la idea de que un derecho racional más allá de toda positivación jurídica es una abstracción vacía y sin fuerza alguna jurídica en la realidad. También ha quedado esclarecida la doble necesidad de la positivación de dichos derechos por lo que respecta a las garantías de su cumplimiento como por lo que respecta a la necesaria concretización de los mismos. Ahora bien, todo ello no contradice ni pone en cuestión el valor suprapositivo y normativo de los principios del derecho racional para configurar la realidad social en la que han de interactuar seres libres y racionales.

Por lo que respecta al valor suprapositivo de los derechos originarios como principios fundamentales del derecho natural, cabe decir que hay que tomar en serio la terminología empleada por Fichte, en virtud de la cual la idea de unos derechos originarios es al mismo tiempo la idea de que hay unos ciertos derechos que no necesitan ser adquiridos para adquirir su validez normativa. El hecho de que no haya derechos originarios fuera de una determinada comunidad estatal y jurídica no significa, pues, eliminar su función normativa para configu-

29 Sobre la dificultad cognoscitiva que implica el derecho del primer ocupante en Fichte y Kant. Cfr., Thiele y Arrese 2010: 72ss.

$30 \mathrm{GA} \mathrm{I} / 3,424$.

31 En este sentido, Fichte señala que «todo individuo tiene solamente derecho a exigir la legalidad del otro, de ningún modo que éste actúe moralmente» GA I, 3, 425. 
rar cualquier ordenamiento jurídico positivo. Lo que hemos puesto de relieve es que cada uno de los derechos originarios expresa un contenido normativo que todo ordenamiento positivo debe respetar y positivizar. Fichte expresa esta idea cuando dice que cada ordenamiento jurídico no debe ser otra cosa que el derecho natural realizado (als das realisierte Naturrecht) ${ }^{32}$ o cuando afirma que el Estado está sometido a deberes jurídicos negativos: a deberes de omisión (Unterlassungspflichten) como límites negativos que no debe violar ningún ordenamiento jurídico. ${ }^{33}$

Desde este punto de vista se puede reconstruir una doctrina normativa del Estado a partir de la teoría fichteana de los derechos originarios, puesto que los derechos a la libertad, a la libre autodeterminación del propio cuerpo y a la propiedad privada han de estar recogidos y garantizados en todo ordenamiento jurídico positivo. Desde esta lectura preeminentemente iusnaturalista de la filosofía del derecho de Fichte cabe interpretar los derechos originarios como derechos inalienables que deben servir como normas y criterios supremos para la configuración positiva del «derecho privado» y del «derecho público» de todo ordenamiento jurídico. En este sentido, cabe decir que la teoría fichteana de los derechos originarios es una fictio juris, pero una fictio juris con alcance normativo para configurar y desarrollar cualquier ordenamiento positivo.

\section{REFERENCIAS BIBLIOGRÁFICAS}

ARRESE I., H., 2010: «El derecho de coacción como garantía del equilibrio del derecho». En: Fichte Online. Estudios sobre Fichte . E. Acosta (Ed.), EuroPhilosophie / Fichte Online, pp. 69-89

FICHTE, J. G., 1966: Gesamtausgabe der Bayerischen Akademie der Wissenschaften, Friedrich Frommann Verlag, Stuttgart-Bad Cannstatt, 1966.

Geismann, G., 1991: «Fichtes Aufhebung des Rechtsstaates». In: FichteStudien, 3, S. 86-117.

HÖFFE, O., 1989: Politische Gerechtigkeit. Grundlegung einer kritischen Philosophie von Recht und Staat, Suhrkamp, Frankfurt/M.

- 2008b: El proyecto político de la modernidad, FCE., Buenos Aires-México.

HORSTMANN, R-P., 2001: «Theorie des Urrechts (§§ 8-12)», en: Grundlage des Naturrechts. Merle, J-C., (Ed.), Akademie Verlag, Berlín.

KANT, I., 1902: Kant's gesammelte Schriften, hrsg. von der Preussichen und der Deutschen Akademie der Wissenschaften, Berlin, 1902 ss.

KERSTING, W., 2001: Filosofia política del contractualismo moderno. Plaza y Valdés, México.

32 Cfr., GA I/3, 432.

33 Cfr., Thiele 2002: 27.

Suplemento 19 (2014) de Contrastes. Revista internacional de filosofía 
- (2007): Wohlgeordnete Freiheit. Immanuel Kants Rechts- und Staatsohilosophie. Mentis Verlag, Paderborn.

PAPADOPOULUS, T., 1993: Die Theorie des Eigentums bei J. G. Fichte, Ars Una, München.

SERRANO, V., 2008: «Fichte y la compresión de los derechos humanos», en: Fichte et la politique. Christophe G.; Rivera de Rosales, J. (Eds.), Polimetrica, Italia, pp. 211-223.

THIELE, U., 2002: Distributive Gerechtigkeit und demokratischer Staat. Fichtes Rechtslehre von 1796 zwischen vorkantischem und kantischem Naturrecht, Duncker\&Humblot, Berlin

VV.AA (2001): Grundlage des Naturrechts. Merle, J-C. (Ed.), Akademie Verlag, Berlín.

VV.AA. (2008): Fichte et la politique. Christophe Goddard, J.; Rivera de Rosales, J. (Eds.), Polimetrica, Italia.

OSCAR CUBO realiza en la actualidad labores docentes en la Facultad de Filosofía de la Universidad de Tréveris (Alemania) y labores de investigación en el Ebbinghaus-Archiv asociado a dicha Facultad.

Líneas de investigación:

Ha realizado estancias de instigación relacionadas con el idealismo alemán en la Universidad de Buenos Aires y como becario posdoctoral de la Fundación Alexander von Humboldt en las universidades de Tubinga y Tréveris.

Publicaciones recientes:

2010: La actualidad hermenéutica del "Saber absoluto". Una lectura de la Fenomenología del Espíritu de Hegel, Madrid, Dykinson.

2012: Kant. Sentido común y subjetividad, Madrid, PyV.

Dirección electrónica: oscarcug@hotmail.com 